\title{
Astroparticle Physics with H.E.S.S.: recents results and near- future prospects
}

\author{
Emmanuel Moulin ${ }^{1, a}$ for the H.E.S.S. collaboration \\ ${ }^{1}$ CEA, Irfu, Université Paris-Saclay, F-91191 Gif-Sur-Yvette Cedex, France
}

\begin{abstract}
H.E.S.S. is an array of five Imaging Atmospheric Cherenkov Telescopes located in Namibia. It is designed for observations of astrophysical sources emitting veryhigh-energy (VHE) gamma rays in the energy range from a few ten GeVs to several ten TeVs. The H.E.S.S. instrument consists of four identical $12 \mathrm{~m}$ diameter telescopes and a $28 \mathrm{~m}$ diameter telescope placed at the center of the array. An ambitious Astroparticle Physics program is being carried out by the H.E.S.S. collaboration searching for New Physics in the VHE gamma-ray sky. The program includes the search for WIMP dark matter and axion-like particles, tests of Lorentz invariance, cosmic-ray electron measurements, and search for intergalactic magnetic fields. I will present the latest results on dark matter search from the observations of the Galactic Centre region, the search for Lorentz invariance violation with the 2014 flare observation of Markarian 501, and the first measurement of the cosmic-ray electron spectrum up to $20 \mathrm{TeV}$. The future of the H.E.S.S. Astroparticle Physics program will be discussed.
\end{abstract}

\section{Introduction}

Very-high-energy (VHE, $\mathrm{E} \gtrsim 100 \mathrm{GeV}$ ) gamma-ray astronomy has developed as a mature research field over the last few decades. Observations carried out by Imaging Atmospheric Cherenkov telescopes (IACT) enable to pursue rich astroparticle physics programmes to address contemporary fundamental topics such as the quest for dark matter in the form of weakly interacting massive or axion-like particles, tests of Lorentz invariance with the observations of bright and distant sources, the search for intergalactic magnetic fields with the observations of bright and hard spectum blazars, and measurements of the VHE cosmic-ray electrons to probe Galactic emission processes taking place in local astrophysical sources and/or the interstellar medium.

\section{Search for heavy weakly interacting dark matter}

A long-term observation programme is being carried out by the H.E.S.S. collaboration to search for signatures of dark matter (DM). Observations focus on close-by regions in the sky with expected high DM density and low gamma-ray background. The central region of the Milky Way is a prime target for DM detection due to its proximity and large DM density as expected at the minimum gravitational potential well of the Galaxy. However, the Galactic Centre (GC) environment is very complex and

a e-mail: emmanuel.moulin@cea.fr 
numerous VHE gamma-ray emissions are detected that represent a challenging background for DM searches. Among them are HESS J1745-290 coincident with the supermassive black hole Sagittarius $\mathrm{A}^{*}$ [1] and a diffuse emission [2] related to energetic protons accelerated to PeV energies [3]. Alternative targets are nearby dwarf spheroidal galaxies $(\mathrm{dSph})$ : they are composed of old stellar populations and harbor a low gas content, and thus are expected to be free from conventional background. This makes them targets for TeV DM that well complement GC region observations and could provide unambiguous detection of DM signals. However, the expected DM signals are a few orders of magnitude lower than that expected from the GC.

The H.E.S.S. DM strategy is based on the GC region and nearby dSph obsevations to look for VHE gamma rays expected from self-annihilation of heavy weakly interacting massive particles (WIMP). The energy-differential gamma-ray flux expected from the self-annihilation of DM particles of mass $m_{\mathrm{DM}}$ writes as:

$$
\frac{\mathrm{d} \Phi}{\mathrm{d} E_{\gamma}}\left(E_{\gamma}, \Delta \Omega\right)=\frac{\langle\sigma v\rangle}{8 \pi m_{\mathrm{DM}}^{2}} \frac{\mathrm{d} N}{\mathrm{~d} E_{\gamma}}\left(E_{\gamma}\right) \times J(\Delta \Omega), \text { with } J(\Delta \Omega)=\int_{\Delta \Omega} \int_{\operatorname{LOS}} \mathrm{d} s \mathrm{~d} \Omega \rho^{2}(r(s, \theta)) .
$$

$\langle\sigma v\rangle$ is the thermally-averaged velocity-weighted annihilation cross section and $\mathrm{d} N / \mathrm{d} E_{\gamma}\left(E_{\gamma}\right)$ the energy-differential gamma-ray spectrum per annihilation. The second term $J(\Delta \Omega)$ referred to as the $J$-factor, corresponds to the integral of the square of the DM density $\rho$ along the line of sight (LOS) and in the solid angle $\Delta \Omega$. The coordinate $r$ writes as $r=\left(r_{\odot}^{2}+s^{2}-2 r_{\odot} s \cos \theta\right)^{1 / 2}$, where $s$ is the distance along the LOS, and $\theta$ is the angle between the direction of observation and the target. $r_{\odot}$ corresponds to the distance of the observer with respect to the target.
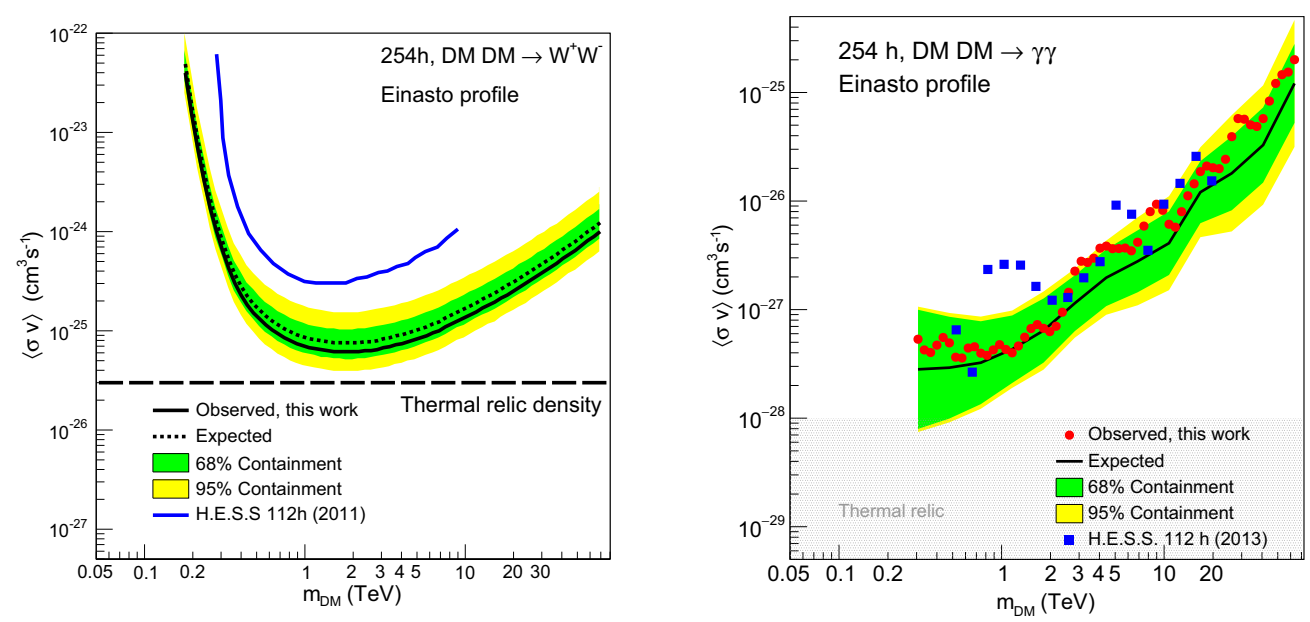

Figure 1. 95\% C.L. upper limits on $\langle\sigma v\rangle$ as function of the DM mass from H.E.S.S. observations of the GC region assuming an Einasto profile for the DM distribution on the GC. The limits are shown for the $\mathrm{W}^{+} \mathrm{W}^{-}$(left) and the prompt $\gamma \gamma$ (right) channels, respectively. In each case, the natural scale expected for $\langle\sigma v\rangle$ is shown. The observed limits together with the expected limits and their associated 1 and $2 \sigma$ containment bands are plotted. Figures extracted from Ref. [4] and Ref. [5], respectively.

The searches led so far in the GC region looked in a region of interest (ROI) of one degree in radius around the GC. The ROI has been defined according to the exposure map obtained from the 
H.E.S.S.-I GC observation strategy and the background extraction technique. All the regions with conventional VHE gamma-ray emissions are excluded [6]. No significant excess signal with respect to background is found. Figure 1 shows the contraints obtained from 10-year H.E.S.S. observations of the GC region. The DM distribution assumes to follow an Einasto profile. The contraints are expressed in terms of $95 \%$ C.L. upper limits on $\langle\sigma v\rangle$ derived from a likelihood test ratio statistics analysis [6]. The observed limits together the expected limit with their 1 and $2 \sigma$ containment bands are shown for DM self-annihilation in the $\mathrm{W}^{+} \mathrm{W}^{-}$(left) and the prompt $\gamma \gamma$ (right) channels, respectively. The limits reach $\langle\sigma v\rangle=6 \times 10^{-26} \mathrm{~cm}^{3} \mathrm{~s}^{-1}$ for a DM mass $m_{\mathrm{DM}}=1.5 \mathrm{TeV}$ in the $\mathrm{W}^{+} \mathrm{W}^{-}$channel [4], and $\langle\sigma v\rangle$ $=4 \times 10^{-28} \mathrm{~cm}^{3} \mathrm{~s}^{-1}$ for $m_{\mathrm{DM}}=1 \mathrm{TeV}$ in the $\gamma \gamma$ channel [5]. They are the most constraining limits obtained so far for TeV DM.

In parallel, H.E.S.S. is leading a long-term observation program of nearby dwarf spheroidal galaxies (dSph) including Sagittarius, the classical dSphs Sculptor, Carina, Fornax, as well as the ultra-faint one Coma Berenices. No VHE gamma-ray emission has been detected so far. The limits reported by H.E.S.S. are summarized in Tab. 1.

Table 1. Upper limits on $\langle\sigma v\rangle$ towards classical and ultra-faint dSphs observed by H.E.S.S. The fourth column provides a round value value of the upper limits at $95 \%$ C.L. extracted from references given in the fifth column.

\begin{tabular}{ccccc}
\hline \hline Target & $\begin{array}{c}\text { Live time } \\
{[\text { hours }]}\end{array}$ & $\begin{array}{c}\text { Annihilation } \\
\text { channels }\end{array}$ & $\begin{array}{c}\text { Upper limits } \\
\text { for } m_{\mathrm{DM}}=1 \mathrm{TeV}\left[\mathrm{cm}^{3} \mathrm{~s}^{-1}\right]\end{array}$ & Refs. \\
\hline \hline Sagittarius & 85.5 & $\mathrm{~W}^{+} \mathrm{W},-\mathrm{ZZ}$ & $2 \times 10^{-23}$ & {$[7]$} \\
& & $\gamma \gamma$ & $5 \times 10^{-25}$ & {$[8]$} \\
Sculptor & 11.8 & $\mathrm{~W}^{+} \mathrm{W},{ }^{-} \mathrm{ZZ}$ & $10^{-22}$ & {$[7,9]$} \\
& & $\gamma \gamma$ & $8 \times 10^{-25}$ & {$[8]$} \\
Carina & 22.9 & $\mathrm{~W}^{+} \mathrm{W}^{-} \mathrm{ZZ}$ & $3 \times 10^{-22}$ & {$[7,9]$} \\
& & $\gamma \gamma$ & $9 \times 10^{-24}$ & {$[8]$} \\
Fornax & \multirow{2}{*}{6.0} & $\mathrm{~W}^{+} \mathrm{W},{ }^{-} \mathrm{ZZ}$ & $8 \times 10^{-22}$ & {$[7]$} \\
& \multirow{4}{*}{10.9} & $\gamma \gamma$ & $3 \times 10^{-24}$ & {$[8]$} \\
Coma Berenices & $\mathrm{W}^{+} \mathrm{W},{ }^{-} \mathrm{ZZ}$ & $2 \times 10^{-21}$ & {$[7]$} \\
& & $\gamma \gamma$ & $8 \times 10^{-25}$ & {$[8]$} \\
Combined & 130 & $\gamma \gamma$ & $5 \times 10^{-25}$ & {$[8]$} \\
\hline \hline
\end{tabular}

\section{Test of Lorentz invariance}

Lorentz invariance is a pillar of special relativity and is established as an exact symmetry up to the precision of current experiments. However, the Lorentz symmetry could be broken at a high-energy scale. In some quantum-gravity (QG) models the foamy structure of spacetime near the Planck scale $\left(\sim 10^{19} \mathrm{GeV}\right)$ could affect high-energy photon propagation in vacuum. One may thus expect a break of the Lorentz invariance at high energies [10]. A phenomenological way to account for possible Lorentz invariance violation (LIV) is to add extra terms in the dispersion relation of photons. The LIV terms are introduced as a series of powers $n$ of the photon energy divided by the QG energy scale $E_{\mathrm{QG}} . n=1$ corresponds to a linear LIV term, while $n=2$ corresponds to a quadratic LIV. The LIV-modified dispersion relation of photons writes :

$$
E \simeq p^{2} c^{2}\left[1 \pm\left(\frac{E}{E_{\mathrm{QG}}}\right)^{n}\right],
$$


where $E$ and $p$ are the energy and momentum of the photon, and the signs + and - corresponds the superluminal and subluminal LIV scenarios, respectively.

The propagation of VHE gamma rays can be affected in two ways: ( $i$ ) the propagation velocity of gamma rays would be dependent on their energy, which would translate into an energy-dependent time delay in the arrival time of gamma rays traveling over astrophysical distances [11]; and (ii) the reaction threshold of the pair-production process on ambient target photons would also be modified as a function of their energy $[12,13]$.

The strong flare of the blazar Markarian (Mrk) 501 in June 2014 has been observed by H.E.S.S. The data show a rapid variability of the flux with VHE gamma rays detected from $\sim 1 \mathrm{TeV}$ up to 20 $\mathrm{TeV}$. These exceptional features have been used to search for LIV-induced effects in the time-of-flight (ToF) and in the spectral measurement approaches. The ToF analysis looked for an energy dependent time delay in the light curve during the flare in two energy ranges: the low-energy one is defined with energies between 1.3 and $3.25 \mathrm{TeV}$, while the high energy one makes use of energies higher than 3.25 TeV. No significant energy-dependent lag in the arrival time of gamma rays is measured between the two regions [14]. The $95 \%$ C.L. lower limits on $E_{\mathrm{QG}}$ reaches $3.6 \times 10^{17} \mathrm{GeV}$ and $8.5 \times 10^{10} \mathrm{GeV}$ for the linear and quadratic terms, respectively. The H.E.S.S. spectrum of the Mrk 501 flare is well fitted

Table 2. Limits on Lorentz Invariance violation from the observations of the 2014 flare of Mrk 501. The constraints are expressed as $95 \%$ C.L. lower limits on $E_{Q G}$ for the linear $(\mathrm{n}=1)$ and quadratic cases $(\mathrm{n}=2)$ in case of subluminal LIV.

\begin{tabular}{ccc}
\hline \hline $\mathrm{n}$ & ToF & Spectrum \\
\hline \hline 1 & $3.6 \times 10^{17} \mathrm{GeV}$ & $2.6 \times 10^{19} \mathrm{GeV}$ \\
2 & $8.5 \times 10^{10} \mathrm{GeV}$ & $7.8 \times 10^{11} \mathrm{GeV}$ \\
\hline \hline
\end{tabular}

by an intrinsic power law attenuated on the EBL using the optical depth as modeled in Ref. [15]. No significant deviation is shown as expected in presence of LIV. Table 2 summarizes the 95\% C.L. constraints on $E_{\mathrm{QG}}$ for the ToF and spectral methods for subluminal LIV. For the spectral method, constraints on $E_{\mathrm{QG}}$ for the linear term reaches $2.6 \times 10^{19} \mathrm{GeV}$, i.e. a factor of two higher than the Planck scale.

\section{Cosmic-ray electron measurements}

Cosmic-ray electrons (CRE) measurements with IACTs convey crucial information on the acceleration of electrons in nearby sources and their propagations in the local interstellar medium. However, only a limited number of nearby accelerators can contribute to the VHE cosmic-ray electron flux measured on Earth given that CREs suffer from strong energy losses during their propagation from inverse Compton scattering on interstellar radiation fields and synchrotron radiation in the $\mu \mathrm{G}$ Galactic magnetic field. CREs of $\mathrm{TeV}$ energies are expected to be produced within about one kpc from the Sun [16]. Among them are the pulsar wind nebulae Gemina, Monogem and Vela, and supernova remnants such as Vela and Vela Junior.

Since the discovery in 2008 by H.E.S.S. of an energy break in the CRE spectrum around 1 $\mathrm{TeV}$ [17], additional observations carried out in extragalactic fields up to 2010 provide now an increased photon statistics in the dataset by a factor of about 4 . In addition to the standard data quality selection criteria, only extragalactic observations with Galactic latitudes $|b|>8^{\circ}$ are used to avoid contamination from Galactic diffuse emission and known VHE gamma-ray sources. Improvements in the data analysis technique based on goodness of fit yield a higher hadron rejection. The H.E.S.S. CRE spectrum is well fitted from $250 \mathrm{GeV}$ up to $25 \mathrm{TeV}$ by a broken power-law function with low 
and high-energy spectral indices of 3.04 $\pm 0.01_{\text {stat }}$ and 3.78 $0.02_{\text {stat }}$, respectively, and an energy break of $\sim 1 \mathrm{TeV}$ [18]. For IACTs, this measurement suffers from systematic uncertainties coming from the event selection cuts involved in the analysis, the zenith angle and year-to-year dependency, as well as the atmospheric conditions of the data taking. A careful estimate of the contamination of misidentified protons in the CRE sample at the highest energies is crucial because of the steeper CRE spectrum compared to that of the protons, i.e. even a small fraction of misidentified protons could induce a spectral hardening that would mimic the contribution of a local source such as Vela or Cygnus loop. Given the present systematic uncertainties, the H.E.S.S. CRE measurement is consistent with both AMS02, CALET, Fermi and DAMPE measurements as well as the recently CRE spectrum measured by VERITAS [19]. The absence of significant spectral features detected in the high-energy end of the spectrum can be used to constrain local source models such as a nearby dark matter clump, local sources such as Vela or Cygnus Loop, and propagation models.

In addition to the spectral CRE measurements, another observable of prime interest to probe local CRE sources is the search for anisotropy in the arrival directions of the CREs. Predictions for the CRE dipole anisotropy in the single source scenario can reach several percents for Vela and Cygnus Loop [20] and a nearby dark matter clump [21]. While such a measurement is challenging for IACTs due to the dependency of the sensitivity to the telescope pointing pattern and the systematic uncertainties arising from the variation of the atmospheric conditions on the CRE flux measurement, no significant dipolar CRE anisotropy has been found by H.E.S.S. 95\% C.L. upper limits for a dipolar anisotropy at the 5\% level are derived for the whole sky [22].

\section{Outlook}

The search for dark matter with H.E.S.S. is crucial to complement the studies done at collider facilities, such as the LHC, or in direct detection experiments. With a sensitivity from the hundred $\mathrm{GeV}$ to the hundred $\mathrm{TeV}$ scale, H.E.S.S. is able to search for heavy weakly interacting DM particles at the TeV scale, a scale which would be difficult to probe at colliders. Since the chance of detection drives the observational strategy, DM searches with H.E.S.S. will focus first on deep observations of the $\mathrm{GC}$ region. A long-term and deep observation program of the inner several degrees of GC is being carried out since a few years in order to achieve the best possible sensitivity before CTA-South comes into operation. Secondly, observations of selected dwarf galaxies will complement those of the GC region. Some of the recently detected dSphs by the Dark Energy Survey (DES) are being observed, their location in the Southern-hemisphere sky guarantees the best sensitivity for H.E.S.S. under the best observational conditions.

LIV-induced effects will be searched both by looking for energy-dependent delay in the arrival time of VHE gamma rays, or by searching for deviations from the standard electron-positron pair production mechanism from the interaction of VHE gamma rays with EBL photons. For the former method, observations of transient or variable sources such as flaring AGNs, pulsars, or GRBs, are required, while for the latter variability is not a prerequisite. Bright and distant sources are a necessity and flaring AGN have been found to be prime objects so far. H.E.S.S. will continue to follow-up on alerts issued by other experiments for flaring AGN or GRBs provided observations are suitable for LIV analyses. Population studies are essential for the near-future LIV searches with astrophysical sources that will become a standard with CTA.

The search for axion-like particles (ALP) that could be detected via their coupling to photons in external magnetic fields will be pursued via observations of distant sources like AGN, where the ALP-photon conversion may occur in the magnetic field of the host galaxy cluster, in the intergalactic magnetic field, as well as in the magnetic field of the Milky Way. While the existence and the strength 
of the intergalactic magnetic field is still elusive, the existence of magnetic fields in galaxy clusters is well established through the observation of radio synchrotron emission as well as through the detection of Faraday rotation in polarized radio sources. ALP-induced effects will be searched through spectral irregularities in AGN emission that may happen from the ALP-photon conversion depending on the strength and morphology of the magnetic field. The most favorable targets are bright AGN located in galaxy clusters for which rotation measures suggest high central magnetic fields.

The search for intergalactic magnetic field will be carried out through the observation of blazars. Following the electron-positron pair production from the interaction of VHE gamma rays with EBL photons, an electromagnetic cascade may develop from the electron/positron interaction with background photons via inverse Compton scattering. In this way, the energy flux from the blazar feeds electromagnetic cascades that may lead to GeV gamma-ray emission. Provided the energy flux remains forward beamed, the level of this $\mathrm{GeV}$ cascade component is commensurate with the amount of the blazars intrinsic spectra absorbed by the EBL. Most favorable candidate sources exhibit both bright emission and hard spectral indices, such as extreme HBL blazars like 1ES 0229+200 and PG $1553+113$.

The H.E.S.S. measurement of the CRE spectrum up to ten TeV energy range enables to probe an uncharted region so far that well complements the satellite measurements carried out with AMS02, CALET, DAMPE. Increased photon statistics in extragalactic fields will help to improve the energy spectrum determination at the highest energies provided that better electron-hadron discrimination is obtained through advanced event separation techniques.

\section{References}

[1] F. Aharonian et al. (H.E.S.S. Collaboration), Astron. Astrophys. 425, L13 (2004)

[2] F. Aharonian et al. (H.E.S.S. Collaboration), Nature 439, 695 (2006)

[3] A. Abramowski et al. (H.E.S.S. Collaboration), Nature 531, 476 (2016)

[4] H. Abdallah et al. (H.E.S.S.), Phys. Rev. Lett. 117, 111301 (2016), 1607.08142

[5] H. Abdallah et al. (H.E.S.S. Collaboration), Phys. Rev. Lett. 120, 201101 (2018), 1805.05741

[6] L. Rinchiuso (for the H.E.S.S. Collaboration), in These proceedings (2018)

[7] A. Abramowski et al. (H.E.S.S. Collaboration), Phys. Rev. D90, 112012 (2014), 1410. 2589

[8] H. Abdalla et al. (H.E.S.S. Collaboration), Submitted to JCAP (2018), 1810.00995

[9] A. Abramowski et al. (H.E.S.S. Collaboration), Astropart. Phys. 34, 608 (2011), 1012 . 5602

[10] G. Amelino-Camelia, Living Rev. Rel. 16, 5 (2013), 0806.0339

[11] G. Amelino-Camelia et al., Nature 393, 763 (1998), astro-ph/9712103

[12] F.W. Stecker, S.L. Glashow, Astropart. Phys. 16, 97 (2001), astro-ph/0102226

[13] U. Jacob, T. Piran, Phys. Rev. D78, 124010 (2008), 0810. 1318

[14] H. Abdalla et al. (H.E.S.S. Collaboration), Submitted to Astrophys. J. (2018)

[15] A. Franceschini, G. Rodighiero, M. Vaccari, Astron. Astrophys. 487, 837 (2008), 0805 . 1841

[16] T. Kobayashi et al., Astrophys. J. 601, 340 (2004), astro-ph/0308470

[17] F. Aharonian et al. (H.E.S.S.), Phys. Rev. Lett. 101, 261104 (2008), 0811.3894

[18] D. Kerszberg et al. (H.E.S.S. Collaboration), in International Cosmic Ray Conference (2017)

[19] A. Archer et al. (VERITAS), Phys. Rev. D98, 062004 (2018), 1808. 10028

[20] S. Manconi, M. Di Mauro, F. Donato, JCAP 1701, 006 (2017), 1611.06237

[21] E. Borriello, L. Maccione, A. Cuoco, Astropart. Phys. 35, 537 (2012), 1012.0041

[22] M. Kraus et al. (H.E.S.S. Collaboration), in International Cosmic Ray Conference (2017) 